\title{
Orientation invariance in naming rotated objects: Individual differences and repetition priming
}

\author{
ELINOR MCKONE and TIM GRENFELL \\ Australian National University, Canberra, Australia
}

\begin{abstract}
In naming drawings of complex common objects, unpracticed naming times increase with rotation away from the upright, but this orientation effect is attenuated with practice. In principle, attenuation could result from learning to extract orientation-invariant information or from learning view-specific representations at the trained orientations. We contrasted these approaches by examining repetition priming for prime-target pairs presented on successive trials in either the same orientation (horse at $51^{\circ}$ primes horse at $51^{\circ}$ ) or a different orientation (horse at $154^{\circ}$ primes horse at $51^{\circ}$ ), for two subgroups of subjects. One subgroup showed no orientation effect, even when unpracticed, and a correspondingly high generalization of priming across different views. The other subgroup initially showed high sensitivity to misorientation and little priming across orientations but, with sufficient practice, came to show no orientation effect and complete generalization of priming. Thus, some subjects always used orientationinvariant procedures, whereas others learned to do so.
\end{abstract}

Object constancy refers to the ability to recognize the identity of an object when it is seen in a variety of different views or orientations. At least three mechanisms have been proposed by which, in principle, object constancy across rotation of an image might be achieved. These include (1) mental rotation to a single stored canonical representation (Cooper \& Shepard, 1973; Shepard \& Metzler, 1971), (2) extraction of orientation-invariant attributes of the object, such as distinguishing features (Jolicoeur, 1985), and (3) storage of multiple representations that are specific to each familiar view (Jolicoeur, 1990; Tarr \& Pinker, 1989). The two theoretical positions that we contrast in the present work both assume that, when an object is presented in an unfamiliar orientation, mental rotation to upright can be used to identify the stimulus. The issue we examine regards the role of the second and third mechanisms in learning with practicethat is, in the process by which an initially novel view eventually becomes as easy to identify as a highly familiar view.

Mental rotation to a canonical view is generally assumed to occur when a familiar object is presented in a novel or an unfamiliar view. For example, when a line drawing of a familiar object with a clearly defined upright is presented (e.g., a horse), reaction times (RTs) to name the object increase progressively as the drawing is rotated in

This research was supported by Grant F96058 from the Australian Research Council and Grant F97072 from the Australian National University. We thank Karl Verfaillie and Asher Koriat for suggestions for improvement on previous versions of this article. Correspondence concerning this article should be addressed to E. McKone, Division of Psychology, Australian National University, ACT 0200, Australia (e-mail: elinor.mckone@anu.edu.au).

$$
\text { -Accepted by previous editor, Myron L. Braunstein }
$$

the picture plane further away from upright in either the clockwise or the counterclockwise direction (Jolicoeur, 1985, 1988; Jolicoeur \& Milliken, 1989; Maki, 1986). This suggests that subjects identify the object in an unfamiliar view by rotating the image until it matches a stored representation of the canonical view, and indeed, Jolicoeur $(1985,1988)$ has shown that the magnitude of the misorientation effect on object naming is consistent with that in a task clearly requiring mental rotation-namely, a judgment regarding whether a stimulus would face left or face right if it were presented upright. (A dip in the orientation function close to $180^{\circ}$ also suggests that some other strategy might be available for stimuli presented upside down.)

Although the effects of misorientation on naming times are large and robust the first time an object is viewed, repeated exposure to the same item gradually attenuates the effect (Corballis, Zbrodoff, Shetzer, \& Butler, 1978; Jolicoeur, 1985; Jolicoeur \& Milliken, 1989; Maki, 1986; Murray, Jolicoeur, McMullen, \& Ingleton, 1993; Tarr \& Pinker, 1989). When the stimuli include only a limited set of a few letters, the misorientation effect disappears completely after only two or so exposures to the set (see, e.g., Corballis et al., 1978). When the stimuli form a larger set of real objects, the misorientation effect endures for longer but is still reduced with practice. For example, Jolicoeur and Milliken presented line drawings in various orientations $\left(0^{\circ}, 60^{\circ}, 120^{\circ}, 180^{\circ}, 240^{\circ}\right.$, and $300^{\circ}$ of rotation clockwise from upright) and repeated the set of objects once in each of six blocks of practice. In the final block, the size of the misorientation effect was more than halved for objects in the trained set, although some effect of misorientation was still apparent. Importantly, the misorientation effect for a new set of objects introduced for the first time in the final block was much larger, showing that whatever was learned with practice was specific to the objects 
studied and did not reflect, for example, a generalized improvement in mental rotation speed.

The question we address concerns what it is that has been learned about the studied objects that attenuates the misorientation effect. Two possible answers to this question have been proposed (Jolicoeur, 1985, 1990; Tarr, 1995; Tarr \& Pinker, 1989; Verfaillie, 1992) - namely, that subjects learn to identify the studied objects via orientation-invariant attributes (e.g., they gradually switch from mental rotation to a feature-based strategy) or, alternatively, that subjects learn additional memory representations specific to each of the trained views (i.e., they add new canonical views).

Tarr and Pinker (1989) have shown that the second of these mechanisms provides a viable route for object identification. They used as stimuli a limited set of novel planar figures, which were highly similar to each other and were deliberately selected to preclude recognition strategies based on unique features. Subjects were taught to associate a name with each object presented in an upright orientation. Objects were then shown in a number of orientations in the image plane. Initially, naming times were dependent on the angular separation of the view tested and the orientation specified as upright, but with sufficient practice, the orientation effect all but disappeared at the trained views. The critical finding was that when new (untrained) orientations were introduced, an orientation effect emerged wherein naming times depended on the angular separation of the novel view from the nearest trained view (i.e., not from that originally defined as upright). This argues that new orientation-specific memory representations were created for the trained views and that Tarr and Pinker's subjects were able to identify a stimulus in a novel view by rotating the image to match the closest of multiple familiar views (see Tarr, 1995, for a similar result with depth rotation of three-dimensional stimuli).

Thus, it appears that, under some circumstances at least, object recognition can be achieved through multiple stored representations of views of the object in a range of orientations. The evidence to date, however, suggests that this mechanism does not underlie the attenuation of misorientation effects when the objects named are line drawings of complex familiar objects, as in the studies of Jolicoeur and his colleagues (e.g., Jolicoeur, 1985; Jolicoeur \& Milliken, 1989; Murray et al., 1993). For example, Jolicoeur and Milliken demonstrated that practice with the specific views then tested is not a precondition for attenuation of the misorientation effect. They trained with one set of objects in the upright view only, intermixed with another set of objects shown in a variety of orientations. Following training, subjects named both sets of objects in a variety of orientations, and the attenuation of the misorientation effect was found to be as strong after practice with only the upright views as it was after practice with mixed views. Murray et al. extended these results by showing that two training trials at one of several orientations reduced the misorientation effect by the same degree on a third exposure regardless of whether the third exposure matched or mismatched the training orienta- tion for that object. They also found that the angular separation between the training and the test orientations had no effect on naming times. Murray (1995) demonstrated that the hypothesis of multiple stored views could not be rescued by arguing that subjects were imagining the appearance of each object in all the possible orientations when only one view was studied, by showing that explicit instructions for subjects to form images of upright objects at specified orientations during training led to no attenuation of the misorientation effect when all the orientations were then presented at test.

Jolicoeur and colleagues have argued that, rather than forming multiple view-specific representations, the subjects in their experiments gradually shifted away from a strategy of identifying objects via mental rotation to the upright and toward a strategy of discriminating between objects in the trained set on the basis of attributes that remained relatively invariant over orientation. Potentially, such attributes might include distinguishing features (e.g., the stripes of a zebra), as well as texture, color, and simple shape attributes (e.g., curves, corners) without knowledge of how these fit together to form the structure of the whole object (Jolicoeur, 1990; Tarr \& Pinker, 1989). McMullen and Jolicoeur (1992) provided support for this hypothesis by contrasting practice effects in the object-naming task (for which a distinguishing feature may be sufficient for identification) with those in a task that, of necessity, requires the extraction of full knowledge of the spatial relationships between different parts of the stimulus. When the subjects judged whether a dot was presented at the top or the bottom of the object (e.g., above the horse's head or below its feet, not at the top or the bottom of the screen), misorientation effects did not diminish with practice, as they did in naming tasks.

Support for the proposal that identification via orientation-invariant features is an optional strategy comes from one aspect of the results of Jolicoeur and Milliken (1989). Although they found that upright views led to normal attenuation of the misorientation effect, this occurred only if these upright views were studied in a mixed list that included other objects presented at a variety of orientations; training on a blocked list in which all the objects were presented only upright did not attenuate the effects of rotation. Thus, the presence of some rotated objects seemed to be necessary to cue subjects to the usefulness of a feature extraction strategy. These results led Jolicoeur (1990) to argue that the use of orientation-invariant attributes is not the default type of information used for pattern recognition but that subjects can rapidly learn to encode and use such attributes when they are available and when attention is drawn to their value.

\section{REPETITION PRIMING, ORIENTATION INVARIANCE, AND ORIENTATION SPECIFICITY}

The present experiments used a short-term repetition priming framework to further examine the reasons that identification of real-world objects becomes insensitive to 


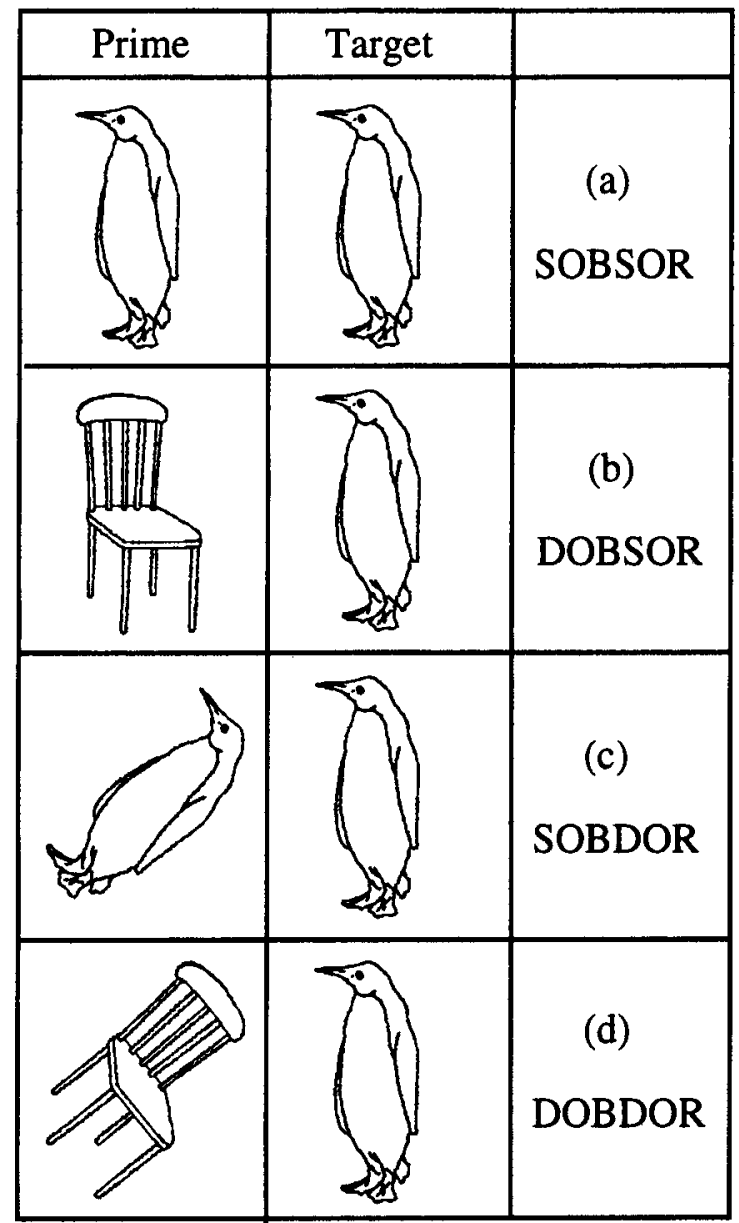

Figure 1. Examples of prime-target pairs in the four conditions used to assess repetition priming - namely, (a) same-objectsame-orientation, or SobSor; (b) different-object-sameorientation, or DobSor; (c) same-object-different-orientation, or SobDor; and (d) different-object-different-orientation, or DobDor. Naming times to targets in DobSor provided the baseline for SobSor (to give $P_{\text {same }}$ ), and DobDor provided the baseline for SobDor (to give $\boldsymbol{P}_{\text {diff }}$ ). Note that the depiction of the target as upright in all cases is for illustrative purposes only; in fact, the target trials depicted objects in all seven orientations.

viewpoint with practice and, particularly, whether it does so via the development of orientation-invariant identification procedures or via the use of multiple view-specific representations. The general approach was to contrast the benefit to naming times accruing from a prior presentation of an object in the same orientation on the previous trial with the benefit accruing from a prior presentation in a different orientation (see Figure 1). Changes in the relative magnitude of different-orientation and same-orientation priming were then examined as a function of practice.

Many previous studies have used the general approach of assessing priming as a function of same versus different stimulus form, using a variety of different types of visual stimuli, including words, line drawings, photographs of three-dimensional objects, faces, and point-light walk- ers. These studies have generally used only two presentations of each stimulus (the prime and the target) and have not examined the development of cross-form priming with practice. In studies of long-term priming (i.e., separate study and test blocks, with prime-target delays of some minutes), unpracticed priming for a repeated word is often greater if the prime and the target have the same appearance than if they differ in form (e.g., Brown \& Carr, 1993; Roediger \& McDermott, 1993; Tenpenny, 1995). In long-term priming for objects, some studies have reported priming to be reduced by depth rotation (Srinivas, 1993) and left-right reflection (Cooper, Schacter, Ballesteros, \& Moore, 1992), although others have found no effects of reflection (Biederman \& Cooper, 1991; Seamon et al., 1997) or of size or color changes (Biederman \& Cooper, 1992; Cooper et al., 1992; Seamon et al., 1997).

Of direct relevance to our design, several studies with objects and faces report that short-term priming (primetarget delays of up to a few trials or a few seconds) is sensitive to rotation, at least to rotation in depth. Marshall and Walker (1987), Roberts and Bruce (1989), and Verfaillie (1993) all report that same-orientation priming is greater than different-orientation priming following depth rotation. This suggests that short-term priming might also be sensitive to plane rotation, at least when subjects are unpracticed with the stimuli.

Our stimuli were of the same type as those employed by Jolicoeur and colleagues-namely, line drawings of familiar complex objects, rotated in the picture plane. The subjects were exposed to 12 blocks of practice with these objects. Within each block, objects occurred in primetarget pairs on successive trials (i.e., the priming of interest was immediate repeat or "lag 0"). On some occasions, the prime trial and the target trial depicted the same object in the same orientation (see Figure 1). On other occasions, the prime trial depicted the same object as the target trial, but in a different orientation. The subjects were required to name each object aloud, and repetition priming was calculated as the increase in naming speed (relative to a baseline where a different object "primed" the target), when the orientation of the prime matched that of the target $\left(\mathrm{P}_{\text {same }}\right)$ or differed from that of the target $\left(\mathrm{P}_{\mathrm{diff}}\right)$.

Given previous findings of orientation specificity in repetition priming, in conjunction with the evidence that subjects initially rotate objects to the upright in order to identify them (i.e., they do not use orientation-invariant procedures), we expected that priming would not generalize fully across orientations when subjects were unpracticed. That is, in the first few blocks of trials, we expected that $P_{\text {diff }}$ would be somewhat less than $P_{\text {same }}$. The question of interest was, then, the extent to which priming would come to generalize across views with repeated exposure. If subjects gradually switch to using a strategy that is invariant across rotation (e.g., feature extraction), $P_{\text {diff }}$ should begin to approach the level of $P_{\text {same }}$ in later blocks and eventually should catch up to it completely. If, in contrast, subjects learn to use view-specific representations 
at the trained orientations, complete generalization of priming across orientation should never occur, no matter how practiced the subjects become, and thus, $P_{\text {diff }}$ should remain well below $P_{\text {same }}$ in all the blocks.

It is, perhaps, worthwhile expanding on the theoretical assumptions leading to these predictions. We make no claims about the exact mechanism by which repetition priming will occur. It is possible that priming might rely on temporary activation of some preexisting representation of each object or on a new short-term memory trace of a recently presented object. We allow that each of these types of representations could be either orientation free or orientation dependent. Our working assumption is merely that (1) if processing of the prime is achieved by orientation-invariant mechanisms, either the representation activated by the prime or the novel trace left by it will include no information regarding orientation and, thus, priming will be orientation invariant, whereas (2) if the prime is identified via orientation-dependent mechanisms, the representations activated or the novel trace left will include information about orientation and, thus, priming will be orientation dependent.

The results to be presented support the interpretation that Jolicoeur and colleagues propose for their data; that is, we show that different-orientation priming can come to match same-orientation priming and, thus, that common objects with distinctive features can be identified on the basis of orientation-invariant representations or procedures. Somewhat accidentally, we also identified substantial individual differences between subjects, both in initial sensitivity to misorientation and in corresponding patterns of priming. These individual differences were so extreme as to suggest a modification of Jolicoeur's (1990) conclusion that the extraction of attributes that are invariant over rotation is, although an optional strategy for object identification, not the default strategy. We report a subgroup of subjects for whom a lack of any misorientation effect at all (i.e., even when unpracticed) argues for a default strategy of identification via orientationinvariant procedures, at least when distinguishing features are available.

\section{EXPERIMENT 1}

\section{Method}

\section{Subjects}

There were 26 subjects, of whom all but one were naive as to the purposes of the experiment. Ten Australıan National University undergraduates participated in return for credit in an introductory psychology course. Sixteen members of the wider university community participated on a purely voluntary basis. All spoke English as their first language and had normal or corrected-to-normal vision.

\section{Design}

Each subject received 12 blocks of trials. Within a block, each object in the set was presented once (for an object unprimed in that block) or twice (for an object prımed in that block). Seven equally spaced orientations were used-namely, $0^{\circ}, 51^{\circ}, 103^{\circ}, 154^{\circ}, 206^{\circ}$, $257^{\circ}$, and $309^{\circ}$ of rotation clockwise from upright. (These orienta- tions were selected so as to avoid the dip in the unpracticed orientation function found close to $180^{\circ}$.) The trials were grouped as prime-target pairs on successive trials; no distinction in procedure was made between prime trials and target trials.

Withın this structure, there were two aspects to the design. In a manipulation check on unprimed trials, orientation $\left(0^{\circ}-309^{\circ}\right)$ was crossed with practice (Blocks 1-12). To address the question of direct interest, priming was then examined by crossing prime-target orientation match (same or different) with practice (Blocks 1-12). All manipulations were made within subjects, and the dependent measure was latency to name the object aloud.

Figure I shows examples of the four conditions used to assess priming (two primed and two baseline). In each case, it was the naming time to the target trial in the pair that was of interest. Priming from an object repeated in the same orientation $\left(\mathrm{P}_{\text {same }}\right)$ was assessed by comparing the same-object-same-orientation (SobSor) condition with a baseline provided by the different-object-sameorientation (DobSor) condition. Priming from an object repeated in a different orientation $\left(P_{\text {diff }}\right)$ was assessed by comparing the sameobject-different-orientation (SobDor) condition with a baseline provided by the different-object-different-orientation (DobDor) condition.

\section{Materials}

The stimuli were made up of 54 line drawings of common objects selected from the Snodgrass and Vanderwart (1980) set. The objects all had a predominant orientation identıfied as $0^{\circ}$ (upright) and included a mixture of natural objects (e.g., a mushroom) and synthetic objects (e.g., a refrigerator). Each drawing was copied in seven orientations from $0^{\circ}$ to $309^{\circ}$ in $51.4^{\circ}$ steps of clockwise rotation in the image plane.

The trial structure was organized as follows. The stimuli were presented in 12 blocks of practice through the full set of objects, with 72 trials per block. These 72 trials included nine prime-target pairs for each of the four priming conditions. Each of the 54 objects appeared unprimed once in each block (i.e., on trials in which the preceding trial depicted a different object), and 18 objects were repeated. The order of conditions was rerandomized for every block and for every subject.

Target trials were then assigned as follows. For any given block for any given subject, 36 objects were randomly selected from the pool of 54 to fill the target trials in each prime-target pair (4 priming conditions $\times 9$ occurrences of each condition $=36$ objects $)$. The orientation in which these objects would be depicted was randomly selected, with the constraint that all seven orientations were used at least once in the nine trials for each priming condition (two orientations were used twice).

After the object identity and orientation had been determined for the target in each prime-target pair, the matching prime trial (i.e., the immediately preceding trial) was filled. In the SobSor condition, the prime trial depicted the same object in the same orientation as the target trial. In the DobSor condition, the orientation of the "prime" matched that of the target, but the identity of the object was randomly selected from the remaining 18 objects in the pool. In the SobDor condition, the prime trial depicted the same object as the target, but its orientation was randomly selected, with the constraint that it differ from that of the target. In the DobDor condition, the objects were the remaining unused items from the pool for that block, and the orientation was randomly assigned, with the constraint that it differ from that of the target.

Note that two unprimed conditions (DobSor and DobDor) were used as baselines against which to assess repetition benefits (for $\mathbf{P}_{\text {same }}$ and $\mathbf{P}_{\text {diff }}$, respectively), rather than a single different-object condition. We chose to use separate baselines so that priming for the identity of an object repeated within and across orientations would not be confounded with a possible generalized effect of priming a 


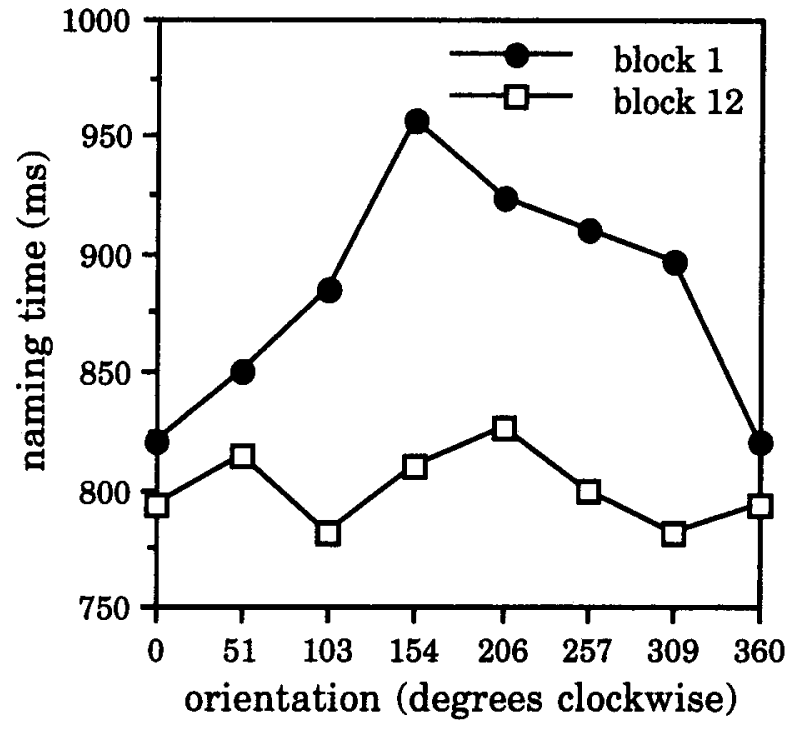

Figure 2. Experiment 1: whole-group data showing object naming times (in milliseconds) as a function of clockwise rotation from upright, in the first and last blocks of practice.

frame of reference that reflected the orientation of the principal axis of the object (Koriat \& Norman, 1984; but see Koriat \& Norman, 1988). As it turned out, this choice of separate baselines was overly cautious: RTs to unprimed objects were found to be only $3 \mathrm{msec}$ faster when the (different) object on the immediately preceding trial matched the target in the alignment of its principal axis than when the alignment was mismatched. Thus, no priming for a frame of reference was apparent.

\section{Procedure}

The subjects were tested individually in a 1-h session. After 10 practice trials (using objects outside the experimental set), the full list of 864 trials was presented three blocks (i.e., 216 trials) at a time. Each group of blocks took approximately $11 \mathrm{~min}$ to complete, and rest breaks were allowed between groups. The stimuli were presented on a PowerMac Macintosh computer, using PsyScope software (Cohen, MacWhinney, Flatt, \& Provost, 1993). The subjects sat approximately $60 \mathrm{~cm}$ from the screen, at which each picture subtended approximately $7^{\circ}$ of visual angle.

The procedure for every trial was exactly the same, regardless of whether that trial depicted a prime or a target: The subject's task was always to name the presented object into a microphone as quickly and as accurately as possible. RTs were recorded as the latency between the onset of the stimulus and the onset of the response. A new trial commenced every $3 \mathrm{sec}$. The object was displayed for a maximum of $2,400 \mathrm{msec}$ but disappeared from the screen when the subject responded. At the end of the $2,400-\mathrm{msec}$ period, a visual distractor was presented for $500 \mathrm{msec}$ that contained rows of $X$ s covering the same area of the screen as the line drawings. The screen was then cleared for $100 \mathrm{msec}$ before the onset of the next trial. Given mean RTs of approximately $800-900 \mathrm{msec}$, these procedures ensured that the average delay between the offset of the object on one trial and the onset of the object of the next was more than $2 \mathrm{sec}, 500 \mathrm{msec}$ of which was filled by the display of $X \mathbf{s}$. This means that any repetition advantage could not be attributed to simple visual persistence from one trial to the next. It also made it unlikely that a backward alignment procedure could operate, by which an object is recognized by rotating its image to match the appearance of the immediately preceding visual stimulus (Koriat, Norman, \& Kimchi, 1991).

\section{Results and Discussion}

Object-naming times were deleted from the analysis if they fell below 300 or above $1,500 \mathrm{msec}(2.8 \%$ of the data) or if the response was in error (3.1\% of the data). Errors included genuine misnamings (e.g., naming a refrigerator as a cupboard), failures to respond, and stuttering, but also included technical problems with the microphone response (e.g., the response was too quiet to trigger the voice key, or the voice key was triggered by an extraneous noise). Most of the $3.1 \%$ error rate was attributable to technical problems. Genuine misnamings occurred on fewer than $1 \%$ of the trials. Although there was some ambiguity in what constituted the ideal name for each object (e.g., fridge vs. refrigerator, or toadstool vs. mushroom), the subjects almost always chose a reasonable name and then used it consistently throughout the experiment. Given the very low rates of genuine mistakes, only RTs (and not errors) were analyzed.

\section{Whole-Group Data}

The RT data were initially analyzed averaged over all 26 subjects. This analysis proceeded in two steps. First, unprimed naming times were examined to confirm that misorientation effects were observed when the subjects were unpracticed and that these effects were reduced over blocks. Second, priming effects were examined as a function of practice to evaluate the contribution of orientationinvariant and view-specific learning to the attenuation of the misorientation effect.

Misorientation and practice effects. The basic effect of misorientation from the upright was evaluated using all unprimed trials in each block-that is, all trials on which the immediately preceding trial did not depict the same object. There were 54 such trials per block. For each of these, the orientation of the object was randomly selected, giving an average per subject of 7-8 unprimed trials per block at each of the seven orientations.

Figure 2 shows mean unprimed naming times in Blocks 1 and 12 as a function of orientation. A substantial misorientation effect was apparent when the set of objects was seen for the first time. In Block 1, naming times increased in an approximately linear fashion as the object was rotated further from the upright in either direction (note that we deliberately avoided using orientations close to $180^{\circ}$ ). After 12 blocks of practice, the orientation effect was no longer apparent. To examine the rate of learning, the slope of the orientation function shown in Figure 2 was calculated. For each block and for each subject, two slope coefficients were computed by assuming linear fits to the two halves of the orientation function (using orientations $0^{\circ}, 51^{\circ}, 103^{\circ}$, and $154^{\circ}$ and then orientations $206^{\circ}, 257^{\circ}, 309^{\circ}$, and $0^{\circ}$ ), and their absolute values were averaged. Figure 3 shows the mean of this average slope as a function of block, expressed as the number of mil- 


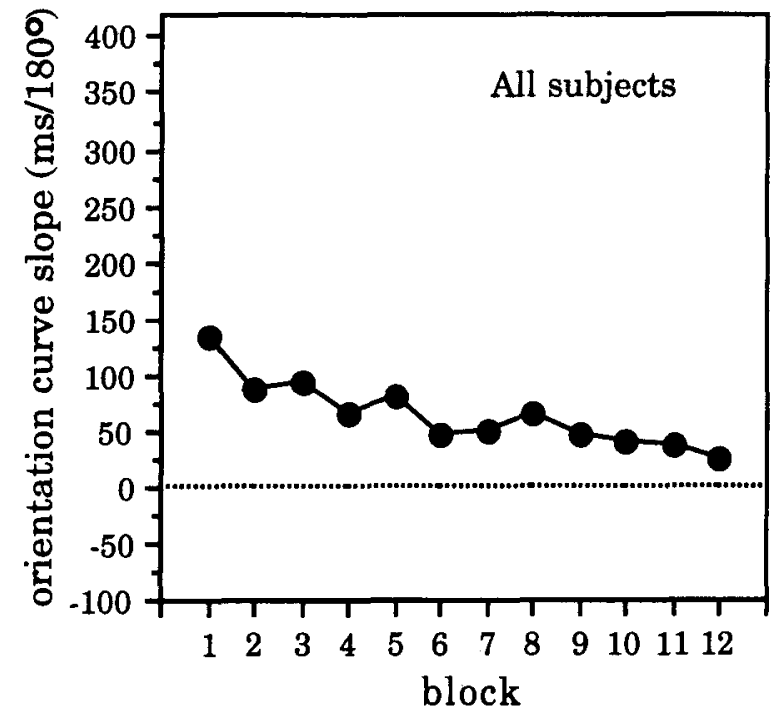

Figure 3. Experiment 1: whole-group data showing the smooth attenuation of the misorientation effect with practice. The size of the misorientation effect is expressed as the number of millisecond increase in naming latency in a putative $180^{\circ}$ of rotation.

liseconds of slowing in naming time in a putative $180^{\circ}$ of rotation. As can be seen, the magnitude of the misorientation effect was reduced smoothly with practice. It should be noted for future reference, however, that the misorientation effect approached zero only in Blocks 11 and 12 , indicating that, on average, the subjects were not fully practiced until the final block or two of the trials.

Statistical analyses confirmed the existence of the orientation effect in the early stages of the experiment and its attenuation with practice. One-way repeated measures analyses of variance (ANOVAs) on the data plotted in Figure 2 revealed a significant effect of orientation in Block 1 [Wilks' lambda $=.370 ; F(6,20)=5.68, M S_{\mathrm{e}}=$ $7,412, p<.005]^{1}$ and no effect of orientation in Block 12 $\left[F(6,168)=1.64, M S_{\mathrm{e}}=4,643, p>.1\right]$. A trend analysis on the attenuation of the orientation effect, shown in Figure 3 , revealed a significant linear trend downward with practice $\left[F(1,25)=8.28, M S_{\mathrm{e}}=25,297, p<.01\right]$. An examination of up to fourth-order trends found no significant higher order components (largest $F=2.70$, smallest $p>.1$ ), which indicates that the orientation effect had not stabilized at zero much before Block 12 (if it had, a quadratic trend should also have been identified).

Baselines and the calculation of priming. Given the confirmation of the standard misorientation effect and its attenuation with practice, the effect of priming an object in the same or a different orientation could then be examined. There were nine trials available per subject per block in each of the two primed and two baseline conditions. The target trials in each of these conditions included only one or two trials at each of the seven orientations, and so the data were collapsed over the actual orientation of the target; the only relevant factor was whether the preceding trial matched the orientation of the target trial or was different from it.

Mean naming times in the baseline conditions (DobSor and DobDor) across Blocks 1-12 were 893, 852, 828, $822,815,811,819,811,794,784,804$, and $807 \mathrm{msec}$, respectively. The two baseline conditions did not differ in any way. As would be expected, baseline RTs decreased over blocks, presumably reflecting a combination of a long-term benefit for individual items repeated across blocks and a generalized improvement in task performance with practice. These effects were particularly strong in the first two to three blocks. The changes in baseline led us to calculate priming within each block in relative terms (e.g., $P_{\text {same }}=($ DobSor - SobSor $) /$ DobSor $\left.\times 100\right)$, rather than as simply the absolute reduction in reaction time (i.e., not as $\mathrm{P}_{\text {same }}=$ DobSor - SobSor). The reason for this was that priming is generally found to be smaller in absolute terms when baseline performance is better and there is less room to improve (e.g., McKone, 1995; Snodgrass, 1989) and a relative measure of priming reduces the influence of these baseline differences.

Same- versus different-orientation priming. Figure 4 shows $P_{\text {same }}$ and $P_{\text {diff }}$ as a function of the 12 blocks of practice, averaged over all 26 subjects. When an object was repeated in the same orientation, priming produced a reduction in naming times of around $25 \%$, which was stable across blocks. When an object was repeated in a different orientation, priming was generally lower. A twoway ANOVA found no main effect of block (Wilks' lambda $\left.=.582 ; F<1, M S_{\mathrm{e}}=100\right)$ and a significant main effect of same- or different-orientation priming $[F(1,25)=$ $\left.22.47, M S_{\mathrm{e}}=259, p<.001\right]$. The more interesting question is whether there was any interaction between orientation match or mismatch and block number, particularly such that $P_{\text {diff }}$ approached $P_{\text {same }}$ with sufficient practice. The whole-group data shown in Figure 4 do not provide convincing evidence for any such catch-up in differentorientation priming, and the ANOVA found no suggestion of an interaction $\left[F(11,275)=1.24, M S_{\mathrm{e}}=63, p>\right.$ .2]. On the other hand, it should be recalled that the unprimed data indicated that it was only by Blocks 11 and 12 that subjects had, on average, received sufficient practice to abolish the orientation effect. Given the lack of fully practiced blocks across which to compare $P_{\text {same }}$ and $P_{\text {diff }}$, it could be argued that Figure 4 does not provide convincing evidence against a catch-up in different-orientation priming.

The results presented in Figure 4 do not lead to a strong conclusion either way about the generalizability of priming across orientations with practice, primarily because not enough data points were available after subjects became fully insenstive to misorientation. ${ }^{2}$ We dealt with this problem in two ways. First, we increased the amount of practice from 12 blocks to 36 blocks for 2 subjects. The results of this procedure are presented shortly as Experiment 2. Second, we reanalyzed the data from Experi- 


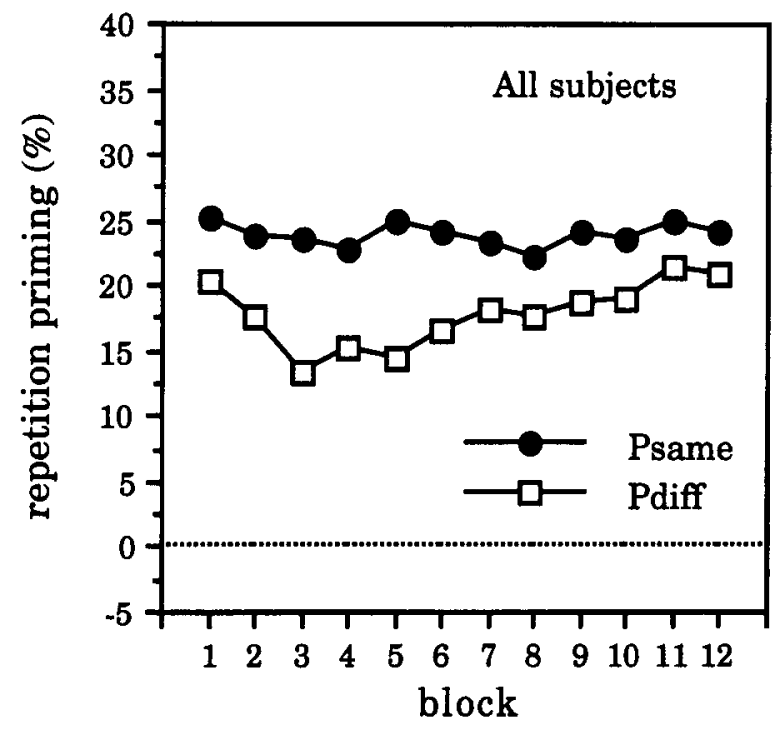

Figure 4. Experiment 1: whole-group data comparing priming between two same-orientation views of the same object $\left(P_{\text {same }}\right)$ and two different-orientation views of the same object $\left(P_{\text {diff }}\right)$, as a function of practice.

ment 1 , taking into account individual differences in sensitivity to orientation. The results of this procedure are presented below.

\section{Orientation-Sensitive and \\ Orientation-Insensitive Subgroups}

Individual differences in orientation sensitivity are commonly reported with the Shepard and Metzler (1971) type of mental rotation tasks, in which subjects must judge whether two simultaneously presented shapes shown from different viewpoints depict the same object or mirror reflections of each other, or must judge whether a shape would face left or right if it had been presented upright. In these types of tasks, some subjects are found to be more strongly influenced in their decision times by the degree of mismatch in orientation than are others (see, e.g., Egan, 1978; Mumaw, Pellegrino, Kail, \& Carter, 1984). It seemed likely that similar individual differences in sensitivity to misorientation might exist for the objectnaming task used here. If this were the case, we felt, it should be possible to pull out data from those subjects in Experiment 1 who were less sensitive to orientation to begin with. Ideally, these subjects might have become fully practiced prior to only the last two blocks of the trials and, thus, could provide more relevant data on whether $P_{\text {diff }}$ can catch up to $P_{\text {same }}$ with sufficient learning.

Of the 26 subjects in the whole group, we extracted data for the 5 subjects who showed the greatest sensitivity to misorientation when unpracticed (orientation-sensitive subgroup) and the 5 subjects who showed the least sensitivity to misorientation when unpracticed (orientationinsensitive subgroup). ${ }^{3}$ There was a high degree of variability in individual-subject data over single blocks, so we determined unpracticed orientation sensitivity by averaging slopes of the orientation curves across the first three blocks of trials. Over these blocks, the 5 most sensitive subjects averaged a slowing in naming times with misorientation of $341 \mathrm{msec} / 180^{\circ}$ (range, 125-500), whereas the 5 least sensitive subjects averaged a misorientation effect of $-6 \mathrm{msec} / 180^{\circ}$ (range, -60 to 19 ). These two groups did not differ noticeably in other ways. Most important, the subjects most sensitive to misorientation were not simply poor at the task overall. In fact, the mean time taken to name upright objects was actually somewhat faster for this subgroup ( $766 \mathrm{msec}$ ) than for the least sensitive subjects ( $822 \mathrm{msec}$ ), although this was not a significant difference $[t(8)=0.80, p>.2]$. (Across all 26 subjects, the mean time to name upright objects was $789 \mathrm{msec}$, $S D=90 \mathrm{msec}$.)

Misorientation and practice effects. Figure 5 plots the orientation curve slopes for the two extreme subgroups as a function of the 12 blocks of practice. Substantial differences in pattern are apparent. Those subjects who began by demonstrating a strong sensitivity to misorientation from the upright gradually became insensitive to rotation with practice, as would be expected from the standard whole-group practice effect. A trend analysis on the slopes for these subjects revealed a significant linear trend downward $\left[F(1,4)=10.42, M S_{\mathrm{e}}=50,208, p<.05\right]$ and no higher order trends (largest $F=1.39$, smallest $p>.2$, up to the fourth order). The most striking aspect of the data, however, is the complete orientation insensitivity of the least sensitive subgroup. Taking into account the ap-

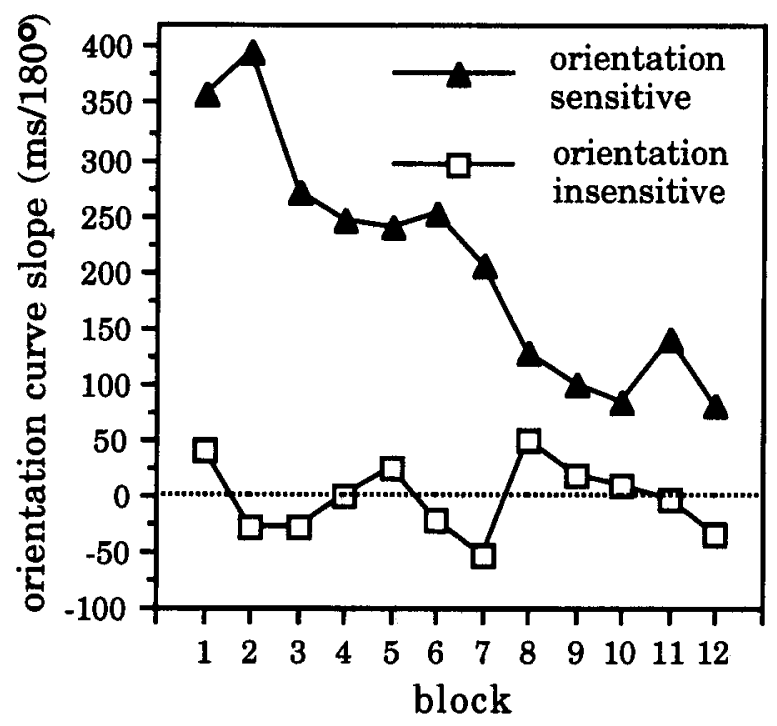

Figure 5. Experiment 1: the size of the misorientation effect with practice for the two extreme subgroups-namely, the 5 subjects most sensitive to orientation in the first three blocks and the 5 subjects least sensitive to orientation in the first three blocks. Note that the orientation-insensitive subgroup shows no effect of misorientation at all, even when unpracticed. The scale is the same as that for the whole-group data in Figure 3. 

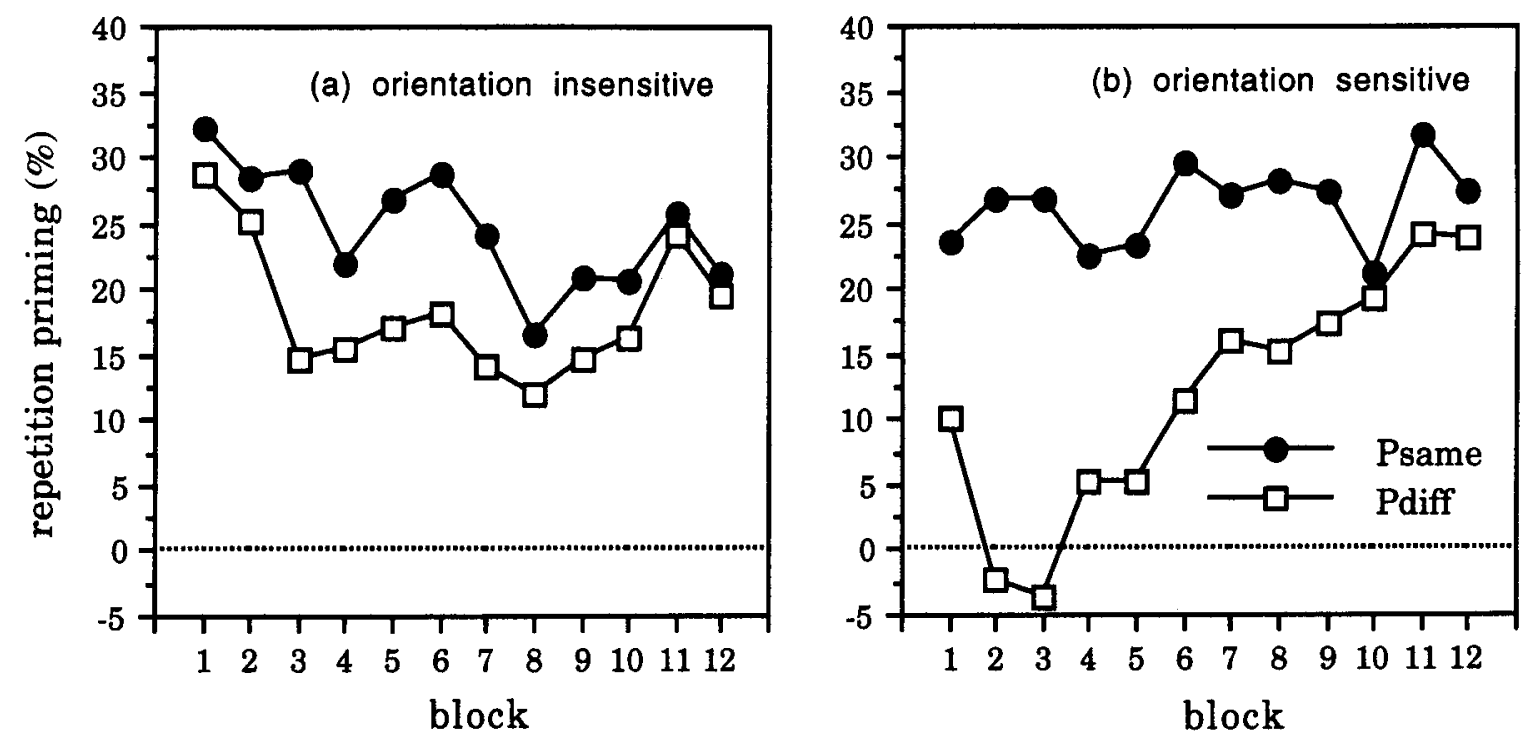

Figure 6. Experiment 1 : comparison of priming for same-orientation and different-orientation views, for (a) the orientation-insensitive subgroup and (b) the orientation-sensitive subgroup. Note that the orientation-insensitive subjects show strong priming between different orientations in all the blocks, whereas orientation-sensitive subjects only achieve this with practice. The scale is the same as that for the whole-group data in Figure 4.

parent error variability evidenced across blocks, this subgroup showed no slope on their orientation function even when unpracticed. This conclusion was supported by the absence of any linear trend in slope values $\left(F<1, M S_{\mathrm{e}}=\right.$ 1,370 ) and the absence of any higher order trends (largest $F=1.83$, smallest $p>.2$, up to the fourth order). The fact that the least sensitive subjects showed smaller misorientation effects when unpracticed than did the most sensitive subjects is, of course, not surprising: This is simply how the groups were defined. What is surprising is that they showed no misorientation effects at all.

Although individual differences in unprimed naming times were not the original focus of this experiment, the differences observed, in fact, provide one of the most interesting results and clearly support the hypothesis that flat orientation curves can reflect the extraction of information that is invariant over rotation. The hypothesis that the subjects learned view-specific representations at the trained orientations cannot explain the fact that some subjects showed flat orientation functions even in Blocks 1-3. In these blocks, the subjects had received no or little exposure to the objects in multiple orientations and, so, would have had no opportunity to learn view-specific representations.

Same- versus different-orientation priming. When $P_{\text {same }}$ and $P_{\text {diff }}$ were examined for each subgroup of subjects, further support emerged for the use of orientationinvariant procedures. Given that the unprimed data suggest that the orientation-insensitive subjects are able to name objects on the basis of orientation-free information even when unpracticed, we would expect that they should, in all the blocks, show a strong generalization of priming across different views of the same object. Figure $6 \mathrm{a}$ shows exactly this pattern. Although $P_{\text {diff }}$ is consistently slightly smaller than $\mathrm{P}_{\text {same }}$ (by approximately $5 \%-10 \%$ ), the difference between same-orientation and differentorientation priming does not change with practice. A twoway ANOVA found a main effect of priming condition that approached significance $\left[F(1,4)=5.75, M S_{\mathrm{e}}=213\right.$, $p=.075]$ and a significant main effect of block, representing a small decline with practice in priming from a single presentation $\left[F(11,44)=2.80, M S_{\mathrm{e}}=70, p<.01\right] .{ }^{4}$ Most important, however, there was no suggestion of any interaction between block and priming condition $(F<1$, $\left.M S_{\mathrm{e}}=54\right)$.

For the subjects who were initially sensitive to misorientation, Figure $6 \mathrm{~b}$ shows a very different pattern of priming. When these subjects were unpracticed, $P_{\text {diff }}$ was small and well below $\mathrm{P}_{\text {same }}$, consistent with the unprimed data showing orientation sensitivity. As these subjects became practiced, however, $P_{\text {diff }}$ gradually caught up to $P_{\text {same }}$ until, by Block 10 or so, priming generalized almost completely across orientations. A two-way ANOVA found a significant main effect of priming condition $[F(1,4)=$ $11.02, M S_{\mathrm{e}}=565, p<.03$ ] and no main effect of block $\left[F(11,44)=1.28, M S_{\mathrm{e}}=217, p>.2\right]$. Most important, there was a significant interaction between block and priming condition $\left[F(11,44)=2.10, M S_{\mathrm{e}}=96, p<.05\right]$.

Angular separation between prime and target. As calculated above, $P_{\text {diff }}$ was produced by collapsing across all the trials for which the prime and the target differed in orientation, without reference to the angular separation 

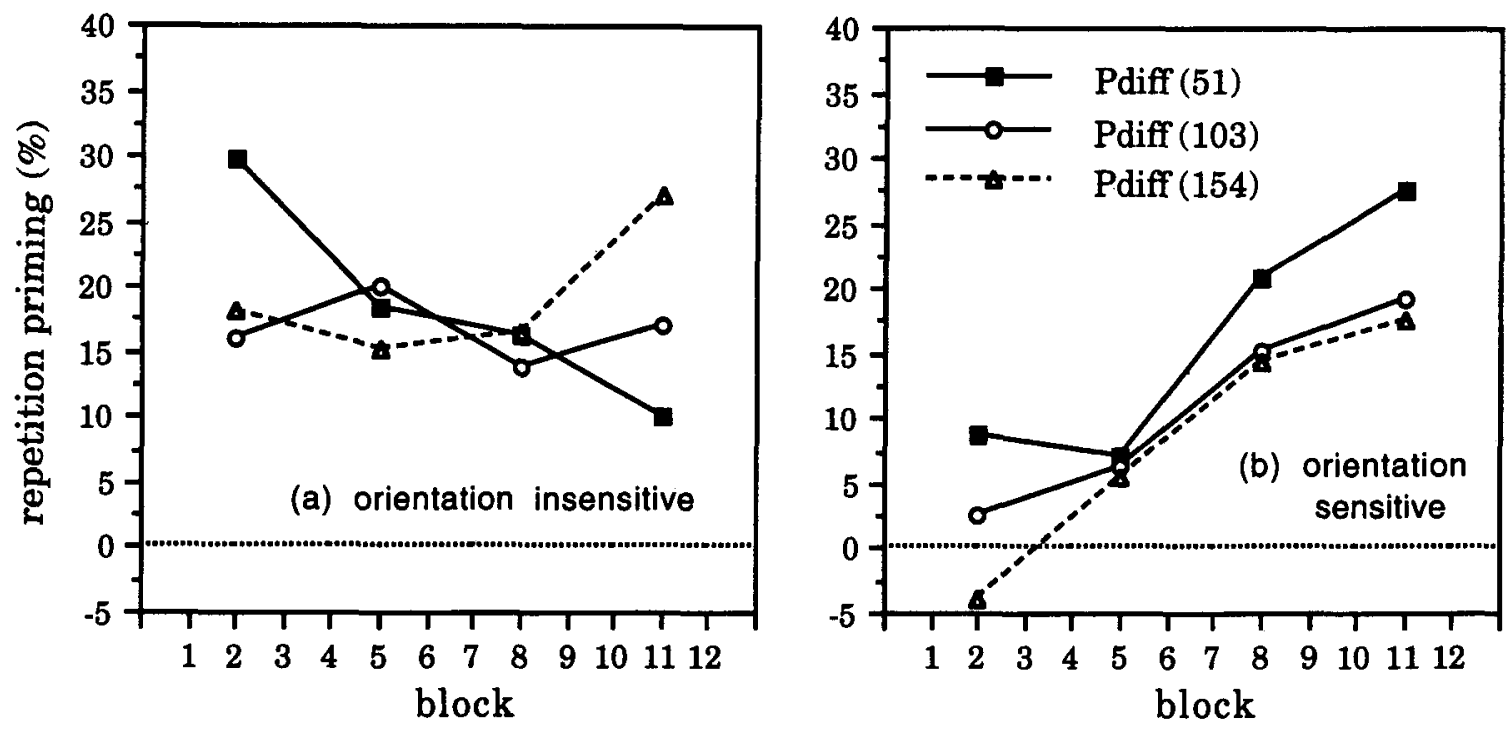

Figure 7. Experiment 1: priming in the different-orientation condition as a function of the angular separation between the prime and the target $\left(51^{\circ}, 103^{\circ}\right.$, or $154^{\circ}$ of rotation in either direction), collapsing the data three blocks at a time. Priming is shown for (a) the orientation-insensitive subgroup and (b) the orientation-sensitive subgroup.

between them. On any given prime-target pair, this angular separation could have been $51^{\circ}, 103^{\circ}$, or $154^{\circ}$, allowing for rotation in either the clockwise or the counterclockwise direction. Analyzing different-orientation priming as a function of the actual degree of separation is of some interest, because object identification via orientationspecific mechanisms should produce progressively less priming as the two views are rotated further away from each other, whereas object identification via orientationinvariant mechanisms should not be affected by the degree of mismatch in views.

Post hoc, the different-orientation trials were split into the three possible angular separation conditions, $\mathrm{P}_{\text {diff }}(51)$, $P_{\text {diff }}(103)$, and $P_{\text {diff }}(154)$. Given that the differentorientation priming condition provided only nine trials per subject per block to be spread among these angular separations and that the random selection of actual angular separation meant that individual subjects had only one or two trials per block in some conditions, we chose to collapse the data three blocks at a time (i.e., Blocks $1-3$, Blocks 4-6, etc.) in order to produce means of approximately the same reliability as those we obtained when comparing $\mathrm{P}_{\text {same }}$ and $\mathrm{P}_{\text {diff }}$ (see Figure 6).

Figure 7 shows different-orientation priming as a function of the angular separation between prime and target trials, for the orientation-insensitive and orientation-sensitive subgroups. The interaction between block and angular separation was not significant in either case ( $p$ s $>.1)$, but interesting differences emerged in the main effects. For the orientation-insensitive subjects, there was no consistent effect of the degree of difference in views, and a twoway ANOVA on the data in Figure 7a found no main effect of angular separation $\left(F<1, M S_{\mathrm{e}}=9,895\right)$. This is what would be expected, given the previous evidence that these subjects were naming the stimuli via orientationinvariant mechanisms. For the orientation-sensitive subjects, in contrast, priming was consistently strongest between views differing only by $51^{\circ}$ and consistently weakest between views differing by $154^{\circ}$, and a two-way ANOVA on Figure $7 \mathrm{~b}$ did find a significant effect of angular separation [Wilks' lambda $=.034 ; F(2,3)=42.8, M S_{\mathrm{e}}=$ $13,758, p<.01]$. This is congruent with the previous evidence that these subjects were, at least when unpracticed, using orientation-specific mechanisms to identify the objects.

\section{Summary}

The results of Experiment 1 indicate that the attenuation of misorientation effects with practice arose from the extraction of orientation-invariant information, not from the learning of view-specific representations at the trained orientations. Support for this interpretation came from four sources. First, the 5 subjects who were least sensitive to misorientation showed no orientation effect on unprimed naming times, even when they were unpracticed and, thus, had had no opportunity to learn orientationspecific views. Second, these subjects simultaneously demonstrated nearly complete transfer of priming across different views of the same object, whereas, in contrast, the 5 subjects who were most sensitive to misorientation showed substantially less priming between two different views than between two same-orientation views. Third, those subjects who were initially sensitive to misorientation eventually came to show strong transfer of priming across different views, and the rate of learning of this transfer corresponded to the rate of attenuation of the 


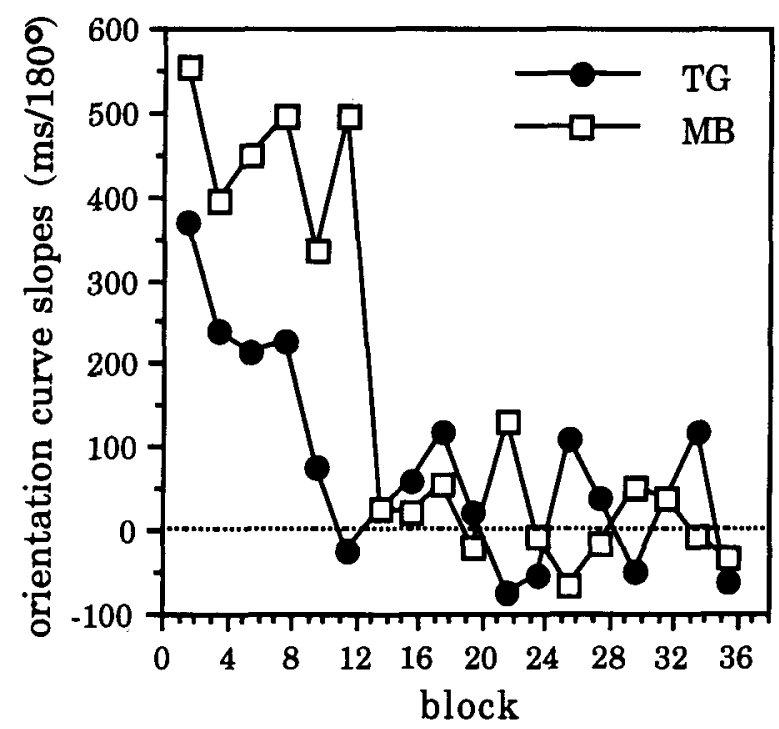

Figure 8. Experiment 2: the size of the misorientation effect for 2 individual subjects, T.G. and M.B., as a function of 36 blocks of practice. The subjects were selected as extreme cases from the orientation-sensitive subgroup of Experiment 1.

misorientation effect on unprimed responses; thus, it was the sensitivity to misorientation in particular blocks that determined whether generalization of priming across different views occurred in that block, regardless of whether that subject demonstrated a natural sensitivity to orientation when unpracticed. Finally, increasing angular separations between the prime and the target in the different-orientation condition reduced priming for the orientation-sensitive subjects but did not do so for the orientation-insensitive subjects.

\section{EXPERIMENT 2}

In Experiment 1, 12 blocks of practice were used. Under this regime, the subjects who were initially sensitive to misorientation from the upright became highly practiced (i.e., the slope of their orientation curves approached zero) only in the last block or 2 of the trials. When priming between and within orientations was assessed for these subjects, it was found that $P_{\text {diff }}$ increased with practice and approached $\mathrm{P}_{\text {same }}$ in the final blocks. It was, however, difficult to know whether $P_{\text {diff }}$ caught up completely to $\mathbf{P}_{\text {same }}$, given that data were available from only 1 or 2 highly practiced blocks. Failure to find a statistically significant difference between $P_{\text {diff }}$ and $P_{\text {same }}$, for example, would be rather unconvincing evidence that $P_{\text {diff }}$ had attained the level of $P_{\text {same }}$, given the small number of subjects involved and the small number of relevant blocks. Thus, the aim of Experiment 2 was to determine whether, with sufficient practice, subjects who initially showed orientation sensitivity would come to show complete generalization of priming across orientations. To address this question, we took 2 subjects who were found, in Experiment 1, to be highly sensitive to misorientation and gave them a further 24 blocks of practice (making 36 blocks in total).

\section{Method}

\section{Subjects}

Two subjects from the orientation-sensitive subgroup of Experiment 1 were selected for further testing. Both had shown very strong effects of misorientation when unpracticed, and both were willing to participate in a further $2 \mathrm{~h}$ of testing on a purely voluntary basis. One subject (M.B.) was naive as to the purposes of the experıment, whereas the other (T.G.) was not.

\section{Materials and Procedure}

The design, materials, and procedure were exactly the same as those for Experiment 1, except that the subjects received 36 blocks of practice in total. The additional 24 blocks of practice were presented in two l-h sessions on successive days ( 12 blocks per day), with the second session conducted the day after Experiment 1 .

\section{Results and Discussion}

Raw RTs were screened for outliers and errors as described in Experiment 1. Error rates for the 2 individual subjects discussed here were no higher than the average for all the subjects in Experiment 1 , and the rate of genuine misnamings was less than $1 \%$ in both cases. Both of the subjects demonstrated extreme sensitivity to orientation when unpracticed. When the slopes of their orientation curves were averaged across the first three blocks of trials, the magnitude of the misorientation effect was $333 \mathrm{msec} / 180^{\circ}$ for T.G. and $477 \mathrm{msec} / 180^{\circ}$ for M.B. Their naming times to upright objects, however, were well within the normal range. Averaged across Blocks 1-12, mean naming times at $0^{\circ}$ were $760 \mathrm{msec}$ for T.G. and $778 \mathrm{msec}$ for M.B., which compared favorably with a whole group mean in Experiment 1 of $789 \mathrm{msec}(S D=$ $90 \mathrm{msec}$ ).

In general, the data in Experiment 2 were examined by collapsing across pairs of successive blocks (i.e., Blocks 1 and 2 were combined, Blocks 3 and 4 were combined, etc.). This was done because only a relatively small number of RTs were available in each condition from a single block (seven to eight per block per subject for unprimed naming times, and nine per block per subject for each of the priming conditions), which made the individual-subject data rather messy when examined one block at a time.

Misorientation and practice effects. Figure 8 plots the slope of the orientation curve as a function of practice for T.G. and M.B. Both of these subjects took some time to become fully practiced. It was only toward the end of the first session (Blocks 11 and 12) that the slope of their orientation functions approached zero. Of most relevance, however, is the evidence that both subjects did achieve a complete level of practice for at least the last half of the experiment. Thus, a comparison of the final levels of $\mathrm{P}_{\text {same }}$ and $\mathrm{P}_{\mathrm{diff}}$ (to be presented below) is strength- 

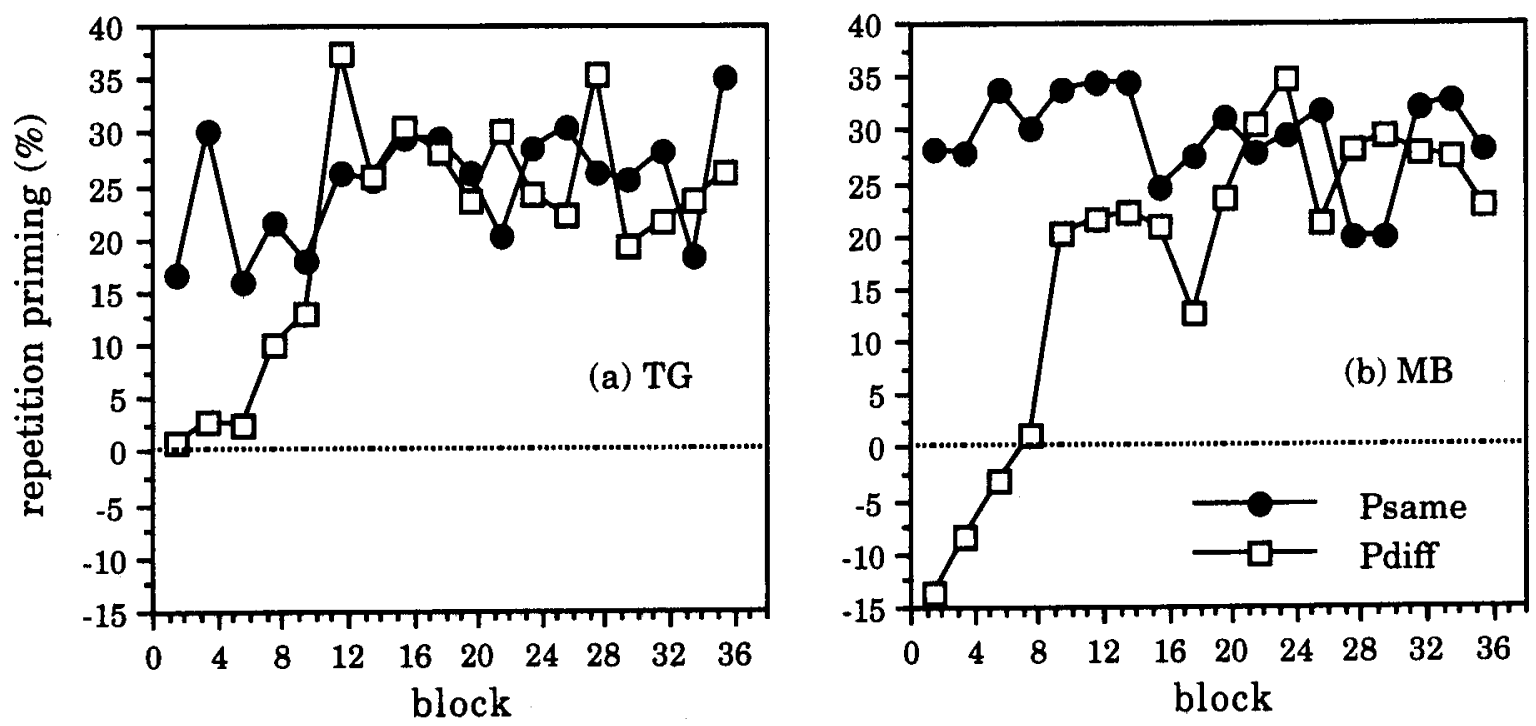

Figure 9. Experiment 2: comparison of priming for same-orientation and different-orientation views across 36 blocks of practice, for (a) T.G. and (b) M.B.

ened by the fact that data were available from at least 15-20 blocks in which each subject demonstrated complete insensitivity to misorientation from the upright.

Same- versus different-orientation priming. Figure 9 shows priming from an object repeated in the same orientation or a different orientation, for T.G. (panel a) and M.B. (panel b). When they were unpracticed, both subjects showed no priming at all between different views of the same object. As they became practiced, however, $P_{\text {diff }}$ climbed consistently until it reached the $P_{\text {same }}$ level for both subjects. To evaluate whether $P_{\text {diff }}$ did, in fact, reach the $P_{\text {same }}$ level, we chose not to conduct significance tests (as these would provide only low power in trying to prove a null hypothesis) or to consider the standard errors of the means (as these reflect only the likely or presumed reliability of each mean). Instead, we selected, as the strongest method of evaluating the difference between $P_{\text {same }}$ and $P_{\text {diff }}$, a simple comparison of this difference with the range of means actually obtained across blocks when general trends had stabilized (as this scatter directly reflects the reliability of the means). For T.G., $P_{\text {diff }}$ and $P_{\text {same }}$ overlapped completely for the last 13 points plotted (i.e., 26 blocks). For M.B., they overlapped completely for the last 9 points plotted (i.e., 18 blocks). These patterns demonstrate the reliability of the lack of any difference between same-orientation and different-orientation priming when the subjects were highly practiced.

Angular separation between prime and target. Figure 10 shows different-orientation priming as a function of the angular separation between the prime and the target (collapsing 3 blocks at a time). Data are presented averaged across subjects T.G. and M.B. The trend toward more priming with smaller separations, which was identified for all five orientation-sensitive subjects in Experiment 1, is apparent in the first 12 or so blocks. It can be seen that, with sufficient practice, however, priming between two views differing by $154^{\circ}$ became as strong as priming between views differing by $51^{\circ}$ or $103^{\circ}$.

Summary. The results of Experiment 2 show that subjects who initially are highly sensitive to misorientation can, with sufficient practice, come to show (1) flat orientation functions, (2) as much priming between two different views of the same object as between two match-

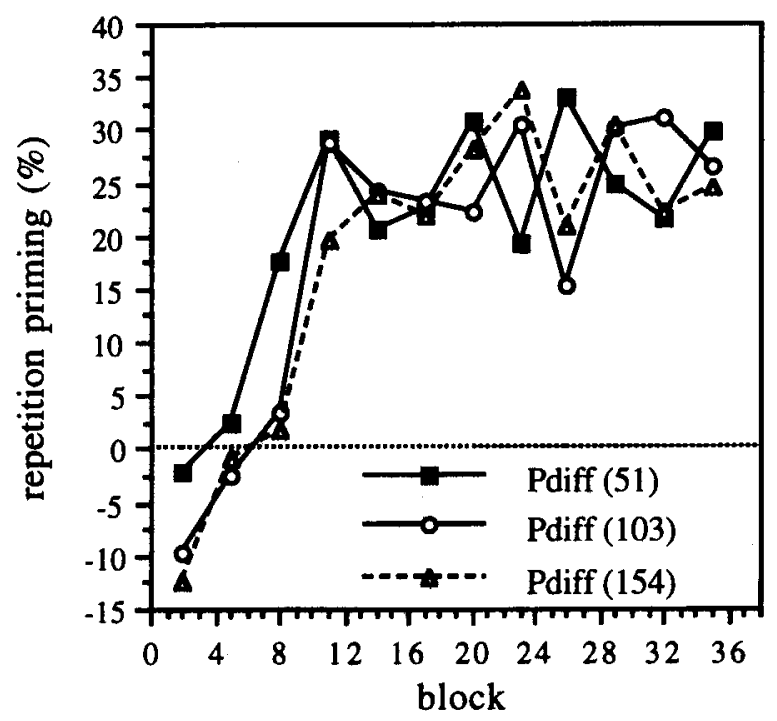

Figure 10. Experiment 2: different-orientation priming as a function of the angular separation between the prime and the target, averaged across T.G. and M.B. 
ing views, and (3) no effect of the angular separation between two different views. That is, these subjects appear able to learn to identify objects via orientation-invariant procedures (or, perhaps, to learn an orientation-invariant memory representation of each object), which can be primed to the same extent by any specific view.

\section{GENERAL DISCUSSION}

The starting point for the present work was to examine two explanations of why misorientation effects on object naming become attenuated with practice - namely, the proposal that subjects learn to recognize orientationinvariant aspects of the objects and the proposal that subjects learn stored orientation-specific representations at each of the trained views. Our data are consistent with the first of these explanations and, furthermore, showed that an orientation-invariant mechanism can operate for some subjects even when they are unpracticed with the stimulus set.

In Experiment 1, substantial individual differences in sensitivity to misorientation from the upright were found. The striking aspect of these individual differences was that the 5 subjects of 26 who were least sensitive to misorientation had completely flat orientation functions, even in the very first blocks of practice. These flat orientation functions cannot be attributed to the learning of orientation-specific views, given that the subjects had not yet been exposed to these views. The use of an orientation-invariant strategy by this subgroup of subjects was confirmed when priming for two same-orientation views $\left(\mathbf{P}_{\text {same }}\right)$ of a given object was compared with priming across two different-orientation views $\left(\mathrm{P}_{\text {diff }}\right)$. Orientation-insensitive subjects showed strong (although not quite complete) generalization of priming across different views, even in Block 1 (i.e., $P_{\text {diff }}$ was nearly as great as $P_{\text {same }}$ ).

The subgroup of 5 subjects who were most sensitive to orientation when unpracticed demonstrated a very different pattern of results. This subgroup showed large effects of misorientation in the first few blocks of practice (naming times were slowed by rotation by at least $100 \mathrm{msec}$ in all cases) and demonstrated a correspondingly weak transfer of priming across orientations. With practice, however, the misorientation effect was substantially reduced, and $P_{\text {diff }}$ began to approach $P_{\text {same }}$. When 2 highly sensitive subjects were selected for a further 24 blocks of practice in Experiment 2, their misorientation effects eventually disappeared altogether, and priming came to show a corresponding complete generalization across orientations.

When different-orientation priming was examined as a function of the degree of angular separation between the prime and the target $\left(154^{\circ}, 103^{\circ}\right.$, or $\left.51^{\circ}\right)$, congruent results were obtained. The orientation-insensitive subjects showed no consistent effect of angular separation, even in the early stages of practice. The subjects who were initially sensitive to misorientation showed less priming at larger prime-target separations when unpracticed, but no effect of angular separation when practiced.

Our results support those of Jolicoeur and Milliken (1989), McMullen and Jolicoeur (1992), Murray (1995), and Murray et al. (1993) in showing that the extraction of orientation-invariant aspects of a stimulus can provide a viable mechanism for object identification and that this mechanism can underlie the attenuation of misorientation effects. Tarr (1995) and Tarr and Pinker (1989), in contrast, have shown that the learning of multiple viewspecific representations can also provide a viable mechanism for identification and can also produce attenuation of misorientation effects. ${ }^{5}$ The obvious difference between the present work and the studies of Jolicoeur, Murray, and colleagues, on the one hand, and of Tarr and Pinker, on the other hand, lies in the nature of the stimuli. Like Jolicoeur and Murray, we used a large set of line drawings of complex familiar objects that were relatively distinctive in terms of component features. Tarr and Pinker used a small set of novel objects deliberately chosen to be very similar in terms of component features and provided more practice with each specific view. Although further empirical work is required to determine exactly which aspects of the stimuli are responsible for the differences in results, a reasonable working hypothesis would be that it is the availability of distinguishing features that affects the identification strategy selected by subjects (Murray et al., 1993).

Thus, it seems that there are multiple routes to object identification, including, at least, mental rotation to a stored upright, matching to a view-specific representation (this may be specific to orientation but may generalize over other surface properties of the stimulus, such as size and brightness; Verfaillie, 1992), and recognition via orientation-invariant attributes. Which of these strategies is employed (or, perhaps, which succeeds first in identifying the object) will depend on the nature of the stimuli to be identified. The type of stimuli used by Tarr and Pinker (1989) and the type of stimuli used in the present research both have analogues in real-world situations: The visual system is sometimes required to make fine discriminations between a limited set of highly similar objects (e.g., recognizing a face or finding a specific car in the carpark) and, in other situations, needs to discriminate between large sets of objects that are more visually distinct (e.g., distinguishing a field of grain, a tractor, a horse, and a tree). Given this, it does not seem surprising that a variety of mechanisms would be available, each of which is more or less suited to different identification situations.

Our results are in agreement with those of Jolicoeur, Murray, and colleagues in showing that, when distinguishing features are available, the preferred strategy to speed object identification is to encode and make use of orientation-invariant attributes of the stimulus, and not to form multiple orientation-specific representations. In fact, our individual-differences data suggest that identification via orientation-invariant attributes can, for some subjects, 
be a more important general strategy even than Jolicoeur (1990) suggested. That is, although Jolicoeur states that feature extraction is not the default mode of operation of the visual system, it appears that it is the naturally preferred strategy for a subgroup of subjects, even when they see stimuli for the first time.

The individual differences we found in initial sensitivity to misorientation could, in principle, arise from two sources. Individual differences in the traditional mental rotation tasks (Cooper \& Shepard, 1973; Shepard \& Metzler, 1971) have generally been presumed to arise from differences in the speed with which subjects are able to rotate an image of the stimulus (e.g., Hock \& Ross, 1975; Juhel, 1990; Mumaw et al., 1984), and this may very well be one source of the individual differences found in our task. However, the observation that some subjects show no effect of misorientation at all cannot be explained in terms simply of speed of rotation (even the fastest mental rotator will still take longer to identify a misoriented stimulus than an upright one). It appears that an additional factor leading to individual differences is the propensity of the subject to identify objects via feature extraction. The suggestion, therefore, is that some subjects bring to the experiment a preference for identifying complex familiar objects by recognizing attributes of the stimulus that are invariant over orientation and do so right from the first presentation of the stimulus set. Other subjects, it seems, learn to use such a "route" with practice.

\section{REFERENCES}

Biederman, I., \& CoOPER, E. E. (1991). Evidence for complete translational and reflectional invariance in visual object priming. Perception, 20, 585-593.

BIEDERMAN, I., \& COOPER, E. E. (1992). Size invariance in visual object priming. Journal of Experimental Psychology: Human Perception \& Performance, 18, 121-133.

Brown, J. S., \& CARR, T. H. (1993). Limits on perceptual abstraction in reading: Asymmetric transfer between surface forms differing in typicality. Journal of Experimental Psychology Learning, Memory, \& Cognition, 19, 1277-1296.

Cohen, J., MacWhinney, B., Flatt, M., \& Provost, J. (1993). PsyScope: An interactive graphic system for designing and controlling experiments in the psychology laboratory using Macintosh computers. Behavior Research Methods, Instruments, \& Computers, 25, 257-271.

Cooper, L. A., Schacter, D. L., Ballesteros, S., \& Moore, C. (1992). Priming and recognition of transformed three-dimensional objects: Effects of size and reflection. Journal of Experimental Psychology: Learning, Memory, \& Cognition, 18, 43-57.

CoOPER, L. A., \& ShePard, R. N. (1973). Chronometric studies of the rotation of mental images. In W. G. Chase (Ed.), Visual information processing (pp. 75-176). New York: Academic Press.

Corballis, M. C., Zbrodoff, N. J., Shetzer, L. I., \& Butler, P. B. (1978). Decisions about identity and orientation of rotated letters and digits. Memory \& Cognition, 6, 98-107.

EGAN, D. E. (1978). Characterizing spatial ability: Different mental processes reflected in accuracy and latency scores (Research Rep. No. 1224). Pensacola, FL: Naval Aerospace Medical Research Laboratory.

Hock, H. S., \& Ross, K. (1975). The effect of familiarity on rotational transformation. Perception \& Psychophysics, 18, 15-20.

JoLICOEUR, P. (1985). The time to name disoriented natural objects. Memory \& Cognition, 13, 289-303.
JoLICOEUR, P. (1988). Mental rotation and the identıfication of disonented objects. Canadian Journal of Psychology, 42, 46 1-478.

JOLICOEUR, P. (1990). On the role of mental rotation and feature extraction in the identification of disoriented objects: A dual-system theory. Mind \& Language, 5, 387-410.

JoliCOEUR, P., \& MiLLIKEN, B. (1989). Identification of disoriented objects: Effects of context of prior presentation. Journal of Experimental Psychology Learning, Memory, \& Cognition, 15, 200-210.

JUHEL, J. (1990). Individual differences in the processing of spatial information. Revue de Psychologie Appliquée, 40, 331-356.

Koriat, A., \& Norman, J. (1984). What is rotated in mental rotation? Journal of Experimental Psychology: Learning, Memory, \& Cognition, 10, 421-434.

Koriat, A., \& Norman, J. (1988). Frames and images: Sequential effects in mental rotation. Journal of Experimental Psychology: Learning, Memory, \& Cognition, 14, 93-111.

Koriat, A., Norman, J., \& Kimchi, R. (1991). Recognition of rotated letters: Extracting invariance across successive and simultaneous stimuli. Journal of Experimental Psychology-Human Perception \& Performance, 17, 444-457.

MAKI, R. H. (1986). Naming and locating the tops of rotated pictures. Canadian Journal of Psychology, 40, 368-387.

MARSHALl, E., \& WALKER, P. (1987). Visual memory for pictorial stimuli in a serial choice reaction-time task. British Journal of Psychology, 78, 213-231.

McKone, E. (1995). Short term implicit memory for words and nonwords. Journal of Experimental Psychology. Learning, Memory, \& Cognition, 21, $1108-1126$.

McMullen, P. A., \& Jolicoeur, P. (1992). The reference frame and effects of orientation on finding the top of rotated objects. Journal of Experimental Psychology: Human Perception \& Performance, 18, 807-820.

Mumaw, R. J., Pellegrino, J. W., Kall, R. V., JR., \& Carter, P. (1984). Different slopes for different folks: Process analysis of spatial aptitude. Memory \& Cognition, 12, 515-521.

MURRAY, J. E. (1995). Imagining and naming rotated natural objects. Psychonomic Bulletin \& Review, 2, 239-243.

Murray, J. E., Jolicoeur, P., McMullen, P. A., \& Ingleton, M. (1993). Orientation-invariant transfer of training in the identification of rotated natural objects. Memory \& Cognition, 21, 604-610.

ROBERTS, T., \& BRUCE, V. (1989). Repetition priming of face recogn1tion in a serial choice reaction-tıme task. British Journal of Psychology, 80, 201-211.

RoEDiger, H. L., III, \& MCDERmott, K. B. (1993). Implicit memory in normal human subjects. In F. Boller \& J. Grafman (Eds.), Handbook of neuropsychology (Vol. 8, pp. 63-131). Amsterdam: Elsevier.

Seamon, J. G., Ganor-Stern, D., Crowley, M. J., Wilson, S. M., Weber, W. J., O'Rouke, C. M., \& MahoneY, J. K. (1997). A mere exposure effect for transformed three-dimensional objects: Effects of reflection, size, or color changes on affect and recognition. Memory \& Cognition, 25, 367-374.

Shepard, R. N., \& Metzler, J. (1971). Mental rotation of threedimensional objects. Science, 171, 701-703.

SNODGRASS, J. G. (1989). Sources of learning in the picture fragment completion task. In S. Lewandowsky, J. C. Dunn, \& K. Kirsner (Eds.), Implicit memory: Theoretical issues (pp. 259-282). Hillsdale, NJ: Erlbaum.

SnOdgrass, J. G., \& VANDERwart, M. (1980). A standardized test of 260 pictures: Norms for name agreement, image agreement, familiarity and visual complexity. Journal of Experimental Psychology Learning, Memory, \& Cognition, 6, 174-215.

SRINIVAS, K. (1993). Perceptual specificity in nonverbal priming. Journal of Experimental Psychology: Learning, Memory, \& Cognition, $19,582-602$.

TARR, M. J. (1995). Rotating objects to recognize them: A case study on the role of viewpoint dependency in the recognition of threedimensional objects. Psychonomic Bulletin \& Review, 2, 55-82.

TARR, M. J., \& PINkER, S. (1989). Mental rotation and orientationdependence in shape recognition. Cognitive Psychology, 21, 233-282. TENPENNY, P. L. (1995). Abstractionist versus episodic theories of rep- 
etition priming and word identification. Psychonomic Bulletin \& Review, 2, 339-363.

VERFAILLIE, K. (1992). Variant points of view on viewpoint invariance. Canadian Journal of Psychology, 46, 215-235.

VerfaiLlite, K. (1993). Orientation-dependent priming effects in the perception of biological motion. Journal of Experimental Psychology' Human Perception \& Performance, 19, 992-1013

\section{NOTES}

1 Throughout this article, multivariate analysis of variance results have been presented when the data violated the sphericity assumption of repeated measures ANOVAs.

2 It should be noted that our levels of practice were higher than those generally used in the literature in this area. Given that Jolicoeur and Milliken (1989) found the size of the misorientation effect for line drawings to be more than halved with 6 blocks of practice, we assumed that 12 blocks here would be sufficient. The mean rate of learning in Experiment 1 may be slightly slower than that in Jolicoeur and Milliken's study, perhaps because we included a larger set of objects.

3. The rationale for selecting the 5 most extreme subjects at each end was to obtain a reasonable amount of data per subgroup, while avoiding washing out any differences in patterns of priming by averaging across subjects with substantially different sensivities to misorientation.
The 16 subjects with intermediate degrees of sensitivity to misorientation did not produce any clear patterns of priming (as with the analysis of all the subjects together).

4. This decline in overall level of priming might reflect the decline in baseline RTs across blocks (i.e., less room to improve with increasing practice). Our relative priming measure might not necessanly remove all the contribution of baselines to priming, given that Snodgrass (1989) argues that priming should be calculated as absolute improvement as a proportion of the maximum possible improvement in performance. To do so requires knowing the mınimum RT that object naming will reach with an infinite number of exposures, and obviously, it is not possible to know this. The procedure we have used overestimates the maximum possible improvement in performance by implicitly assuming that the minimum possible RT is 0 msec. Thus, the effect of changing baselines might not fully be compensated for.

5 . Incidentally, this suggests that an application of our repetition priming technıque to an experiment using Tarr and Pinker's (1989) general paradigm would show priming between different orientations to remain consistently less than same-view priming, even when subjects are highly practiced.

(Manuscript received June 18, 1997; revision accepted for publication September 16, 1998.) 